PROCEEDINGS OF THE

AMERICAN MATHEMATICAL SOCIETY

Volume 139, Number 9, September 2011, Pages 3127-3133

S 0002-9939(2011)10750-0

Article electronically published on February 1, 2011

\title{
THE INCIDENCE CORRESPONDENCE AND ITS ASSOCIATED MAPS IN HOMOTOPY
}

\author{
LUIS E. LOPEZ
}

(Communicated by Brooke Shipley)

\begin{abstract}
The incidence correspondence in the grassmannian which determines the tautological bundle defines a map between cycle spaces on grassmannians. These cycle spaces decompose canonically into a product of EilenbergMacLane spaces. These decompositions and the associated maps are calculated up to homotopy.
\end{abstract}

\section{INTRODUCTION}

Let $\mathcal{C}_{d}^{p}\left(\mathbb{P}^{n}\right)$ denote the set of all effective algebraic cycles of codimension $p$ and degree $d$ in $\mathbb{P}^{n}$. This set can be given the structure of an algebraic variety via the Cayley-Chow-Van der Waerden embedding (see [1], [5]) which takes an irreducible cycle $X$ into $\Psi(X)$, where the Chow Form $\Psi$ is obtained from the following incidence correspondence:

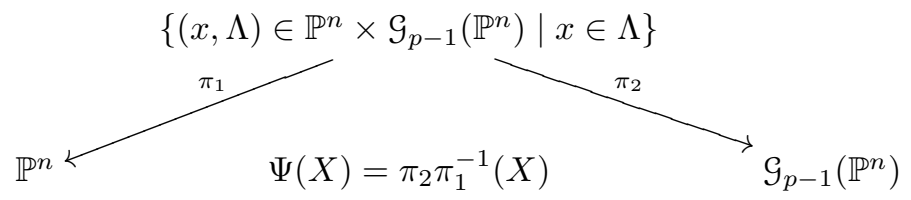

This map is then extended additively to the topological monoid $\mathfrak{e}^{p}\left(\mathbb{P}^{n}\right)$ of all effective cycles and to the topologized Grothendieck group $Z^{p}\left(\mathbb{P}^{n}\right)$. The cycle $\Psi(X)$ has codimension 1 in the grassmannian; therefore $\Psi$ defines a map

$$
\Psi: z^{p}\left(\mathbb{P}^{n}\right) \rightarrow z^{1}\left(\mathcal{G}_{p-1}\left(\mathbb{P}^{n}\right)\right) .
$$

Moreover, if $B=\bigoplus B_{d}$ denotes the coordinate ring of the grassmannian in the Plücker embedding, then every irreducible hypersurface $Z$ of degree $d$ in the grassmannian is given by an element $f \in B_{d}$ defined uniquely up to a constant factor (see 5 ). This defines a grading on the space of hypersurfaces in the grassmannian, and with respect to this grading the map above preserves the degree.

Received by the editors March 4, 2009 and, in revised form, August 16, 2010.

2010 Mathematics Subject Classification. Primary 14C99; Secondary 55R35.

Key words and phrases. Incidence correspondence, Chow varieties, cycles on grassmannians.

The author thanks the Max-Planck-Institut für Mathematik for its hospitality during the writing of this work and the reviewer for improvements and corrections.

(C)2011 American Mathematical Society Reverts to public domain 28 years from publication 
The topology thus inherited defines in turn a topology in the space $\mathfrak{C}^{p}\left(\mathbb{P}^{n}\right)$ of all codimension $p$ algebraic cycles in $\mathbb{P}^{n}$ (cf. [3] for this and other equivalent definitions). The results presented here are of the following type:

Theorem 1.1. The Chow Form map

$$
\Psi: z^{p}\left(\mathbb{P}^{n}\right) \rightarrow z^{1}\left(\mathcal{G}_{p-1}\left(\mathbb{P}^{n}\right)\right)
$$

can be represented with respect to decompositions of the corresponding spaces in the following way:

$$
\prod_{j=0}^{p} K(\mathbb{Z}, 2 j) \stackrel{p}{\longrightarrow} K(\mathbb{Z}, 0) \times K(\mathbb{Z}, 2),
$$

where $p$ is the projection into the first two factors of the product.

Hence, the Chow Form map can be interpreted as the classifying map of a (nontrivial) line bundle in the space of algebraic cycles in $\mathbb{P}^{n}$, and the class of this line bundle generates $H^{2}\left(\mathcal{Z}^{1}\left(\mathcal{G}_{p-1}\left(\mathbb{P}^{n}\right)\right)\right)$.

An equivalent way of looking at the theorem is via the chain of inclusions

$$
\mathcal{G}^{p}\left(\mathbb{P}^{n}\right) \subset \mathcal{Z}^{p}\left(\mathbb{P}^{n}\right) \subset \mathcal{Z}^{1}\left(\mathcal{G}_{p-1}\left(\mathbb{P}^{n}\right)\right) .
$$

The first space classifies $p$-dimensional bundles (not all of them), the second space classifies all total integer cohomology classes in $\bigoplus_{j=0}^{p} H^{2 j}(-; \mathbb{Z})$, and the third space classifies all integer cohomology classes in $H^{0}(-; \mathbb{Z}) \times H^{2}(-; \mathbb{Z})$. The corresponding maps associated to the inclusions are the total Chern class map (see [9]) and the projection into the first two factors.

\section{The Chow Form MaP}

In this section we prove the theorem regarding the Chow Form map using an explicit description of the map. In order to state the results and its proofs we recall some definitions and facts about Lawson Homology. A survey of these and other related results is given in $[6$.

Definition 2.1. Let $X$ be a projective variety. The Lawson Homology groups $L_{p} H_{n}(X)$ of $X$ are defined by

$$
L_{p} H_{n}(X):=\pi_{n-2 p}\left(z_{p}(X)\right),
$$

where $z_{p}(X)$ is the naive group completion of the Chow monoid $\mathfrak{C}_{p}(X)$ of all $p$ dimensional effective algebraic cycles in $X$.

These groups stand between the group of algebraic cycles modulo algebraic equivalence $\mathcal{A}_{p}(X)=L_{p} H_{2 p}(X)$ and the singular homology group $H_{n}(X)=L_{0} H_{n}(X)$. Friedlander and Mazur defined a cycle map between the Lawson Homology groups and the singular homology groups:

$$
s_{X}^{(p)}: L_{p} H_{n}(X) \rightarrow H_{n}(X ; \mathbb{Z}) .
$$

This map will be referred to as the F-M map.

The following results of Lima-Filho [7] will be used throughout the section.

Theorem 2.2 (Lima-Filho, [7). The F-M cycle map coincides with the composition

$$
L_{p} H_{n}(X)=\pi_{n-2 p}\left(\mathcal{Z}_{n-p}(X)\right) \stackrel{e_{*}}{\longrightarrow} \pi_{n-2 p}\left(\mathfrak{Z}_{2 p}(X)\right) \stackrel{\mathcal{A}}{\longrightarrow} H_{n}(X ; \mathbb{Z}),
$$

where $e_{*}$ is the map induced by the inclusion

$$
e: \mathfrak{Z}_{m}(X) \rightarrow \mathfrak{Z}_{m}(X)
$$


of the space of algebraic cycles into the space of all integral currents and $\mathcal{A}$ is the Almgren isomorphism defined in 11. In particular, the F-M cycle map is a natural transformation of functors and is compatible with proper push-forwards and flatpullbacks of cycles.

Theorem 2.3 (Lima-Filho, [7]). If $X$ is a projective variety with a cellular decomposition in the sense of Fulton (i.e., $X$ is an algebraic cellular extension of $\emptyset$ ), then the inclusion

$$
Z_{p}(X) \hookrightarrow \mathfrak{Z}_{2 p}(X)
$$

into the space $\mathfrak{Z}_{2 p}(X)$ of integral currents is a homotopy equivalence

We also recall some facts about the cohomology of the grassmannian. We follow the notation of [4, Chapter 14 .

Definition 2.4. Let $\mathcal{G}_{k}\left(\mathbb{P}^{n}\right)$ be the grassmann variety of $k$-dimensional linear spaces in $\mathbb{P}^{n} \cdot \mathcal{G}_{k}\left(\mathbb{P}^{n}\right)$ is a smooth algebraic variety of complex dimension $d:=(k+1)(n-$ $k)$. The special Schubert classes are the homology classes $\sigma_{m} \in H_{2(d-m)}\left(\mathcal{G}_{k}\left(\mathbb{P}^{n}\right)\right)$ defined by the cycle class of

$$
\sigma_{m}:=\left\{L \in \mathcal{G}_{k}\left(\mathbb{P}^{n}\right) \mid L \cap A \neq \emptyset\right\},
$$

where $A$ is any linear subspace of $\mathbb{P}^{n}$ of codimension $k+m$.

Theorem 2.5. The integral cohomology of the grassmannian $\mathcal{G}_{k}\left(\mathbb{P}^{n}\right)$ is generated as a ring by the Poincaré duals $c_{m}(Q)$ of the special Schubert classes $\sigma_{m}$. These Poincaré duals are the Chern classes of the universal quotient bundle $Q$.

Theorem 2.6. The Chow Form map

$$
\Psi: Z^{p}\left(\mathbb{P}^{n}\right) \rightarrow Z^{1}\left(\mathcal{G}_{p-1}\left(\mathbb{P}^{n}\right)\right)
$$

can be represented with respect to decompositions of the corresponding spaces in the following way:

$$
\prod_{j=0}^{p} K(\mathbb{Z}, 2 j) \stackrel{p}{\longrightarrow} K(\mathbb{Z}, 0) \times K(\mathbb{Z}, 2),
$$

where $p$ is the projection into the first two factors of the product.

Proof. We have the following homotopy equivalences:

$$
Z^{p}\left(\mathbb{P}^{n}\right) \simeq \prod_{j=1}^{p} K(\mathbb{Z}, 2 j)
$$

and

$$
z^{1}\left(\mathcal{G}_{p-1}\left(\mathbb{P}^{n}\right)\right) \simeq K(\mathbb{Z}, 0) \times K(\mathbb{Z}, 2)
$$

Moore's theorem states that for an abelian topological group $G$ there is a homotopy equivalence between $G$ and $\prod_{i} K(G, i)$. Therefore it suffices to compute the homotopy groups of each of these Grothendieck groups of cycle spaces. On the other hand, using Theorem 2.3 and Almgren's theorem, which asserts that

$$
\pi_{i}\left(\mathfrak{Z}_{k} X\right) \cong H_{i+k}(X)
$$

we only need to calculate the homology of $\mathbb{P}^{n}$ and $\left(\mathcal{G}_{p-1}\left(\mathbb{P}^{n}\right)\right)$. The homology of $\mathbb{P}^{n}$ is known to be $\mathbb{Z}$ in all even dimensions from 0 through $2 n$ and zero otherwise. 
The homology groups needed for the second homotopy equivalence are the following: if $d=\operatorname{dim}_{\mathbb{C}} \mathcal{G}_{p-1}\left(\mathbb{P}^{n}\right)$, then

$$
\pi_{i}\left(\mathcal{Z}_{d-1} \mathcal{G}_{p-1}\left(\mathbb{P}^{n}\right)\right) \cong \begin{cases}H_{2(d-1)}\left(\mathcal{G}_{p-1}\left(\mathbb{P}^{n}\right)\right) \cong \mathbb{Z} & \text { if } i=0, \\ H_{2 d}\left(\mathcal{G}_{p-1}\left(\mathbb{P}^{n}\right)\right) \cong \mathbb{Z} & \text { if } i=2, \\ H_{2(d-1)+i}\left(\mathcal{G}_{p-1}\left(\mathbb{P}^{n}\right)\right)=0 & \text { otherwise }\end{cases}
$$

this last calculation being well known for grassmannians (see [2]). Now we use Theorem 2.2 to calculate the induced maps in homotopy. We have the following commutative diagram:

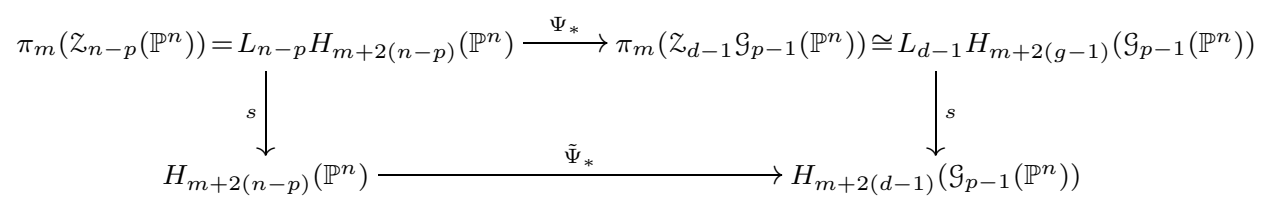

where $\Psi$ is the Chow Form map, $\Psi_{*}$ is the map induced in the homotopy groups of the cycle spaces and $\tilde{\Psi}_{*}$ is the map induced in homology, i.e. $\tilde{\Psi}_{*}=\pi_{2 *} \circ \pi_{1}^{-1}$.

Theorem 2.3 implies that the vertical maps are isomorphisms, and Theorem 2.2 implies that the diagram is commutative since the $s$ map is a natural transformation of functors compatible with push-forwards and pull-backs.

We know the homology of the grassmannian; the only non-zero dimensions in the lower right corner of the diagram correspond to the cases $m=0$ and $m=2$. We will describe explicitly the morphism in these two cases.

$m=0$ In this case $H_{2(n-p)}\left(\mathbb{P}^{n}\right)$ is generated by the class of an $(n-p)$-plane $\Lambda$ in $\mathbb{P}^{n}$. The definition of $\Psi$ says that the cycle $\tilde{\Psi}_{*}(\Lambda)$ is

$$
\tilde{\Psi}_{*}(\Lambda)=\left\{P \in \mathcal{G}_{p-1}\left(\mathbb{P}^{n}\right) \mid \Lambda \cap P \neq \emptyset\right\} .
$$

This is precisely Definition 2.4 of the special Schubert cycle $\sigma_{1}$.

$m=2$ In this case $H_{2+2(n-p)}\left(\mathbb{P}^{n}\right)$ is generated by the class of an $(n-(p-1))$-plane $\Lambda$ in $\mathbb{P}^{n}$. The cycle $\tilde{\Psi}(\Lambda)$ is then

$$
\tilde{\Psi}_{*}(\Lambda)=\left\{P \in \mathcal{G}_{p-1}\left(\mathbb{P}^{n}\right) \mid \Lambda \cap P \neq \emptyset\right\},
$$

but by a dimension count this is the whole variety $\mathcal{G}_{p-1}\left(\mathbb{P}^{n}\right)$ again. This corresponds to the special Schubert cycle $\sigma_{0}$

This completes the proof. An analogous argument works in the general case, which is stated in Theorem 3.2. The strength of Lima-Filho's Theorem 2.2 is remarkable. In the case $m=2$ the top row of the diagram is a map involving a second homotopy group, which seems to disappear magically, being replaced by a simple reasoning using the incidence correspondence and its associated maps in homology. But this is precisely what the theorem is stating: when the $s$ map turns out to be an isomorphism, we can compute what happens upstairs in homotopy using homology calculations from downstairs (this line of reasoning is also used in the proof of Theorem 2.7 below).

The results of Lima-Filho about the F-M cycle class map may also be used to prove the following theorem of Lawson and Michelsohn, which appeared in 9 . 
Theorem 2.7. The complex join pairing in the cycle spaces

$$
\#: Z^{q}\left(\mathbb{P}^{n}\right) \wedge z^{q^{\prime}}\left(\mathbb{P}^{m}\right) \rightarrow z^{q+q^{\prime}}\left(\mathbb{P}^{n+m+1}\right)
$$

represents the cup product pairing in the canonical decompositions

$$
\bigcup: \prod_{s=0}^{q} K(\mathbb{Z}, 2 s) \wedge \prod_{t=0}^{q^{\prime}} K(\mathbb{Z}, 2 t) \rightarrow \prod_{r=0}^{q+q^{\prime}} K(\mathbb{Z}, 2 r) ;
$$

i.e. if $i_{2 c}$ represents the generator of $H^{2 c}(K(\mathbb{Z}, 2 c) ; \mathbb{Z}) \cong \mathbb{Z}$, then

$$
(\#)^{*}\left(i_{2 c}\right)=\sum_{\substack{a+b=c \\ a>0 \\ b>0}} i_{2 a} \otimes i_{2 b} .
$$

Proof. The linear join of two irreducible varieties $X \subset \mathbb{P}^{n}$ and $Y \subset \mathbb{P}^{m}$ with defining ideals $\left\langle F_{i}\right\rangle \in \mathbb{C}\left[x_{0}, \ldots, x_{n}\right]$ and $\left\langle\mathcal{G}_{j}\right\rangle \in \mathbb{C}\left[y_{0}, \ldots, y_{m}\right]$ is the variety in $\mathbb{P}^{n+m+1}$ defined by the ideal $\left\langle F_{i}, G_{j}\right\rangle \in \mathbb{C}\left[x_{0}, \ldots, x_{n}, y_{0}, \ldots, y_{m}\right]$. A synthetic construction of this pairing is given in [9]. The join pairing \# is obtained by extending bi-additively to all cycles and taking the induced pairing in the smash product. The join pairing induces a bilinear pairing

$$
\pi_{s}\left(Z^{q}\left(\mathbb{P}^{n}\right)\right) \times \pi_{r}\left(Z^{q^{\prime}}\left(\mathbb{P}^{m}\right)\right) \rightarrow \pi_{r+s}\left(Z^{q+q^{\prime}}\left(\mathbb{P}^{n+m+1}\right)\right),
$$

which corresponds to a homomorphism

$$
\begin{aligned}
\#_{*}: L_{n-q} H_{s+2(n-q)}\left(\mathbb{P}^{n}\right) & \otimes L_{m-q^{\prime}} H_{r+2\left(m-q^{\prime}\right)}\left(\mathbb{P}^{m}\right) \\
& \rightarrow L_{(n-q)+\left(m-q^{\prime}\right)+1} H_{s+2(n-q)+r+2\left(m-q^{\prime}\right)+2}\left(\mathbb{P}^{n+m+1}\right) .
\end{aligned}
$$

This homomorphism is non-zero only when $s$ and $r$ are even, so we may assume $s=2 a$ and $r=2 b$. Now we take the F-M map and get the following commutative diagram:

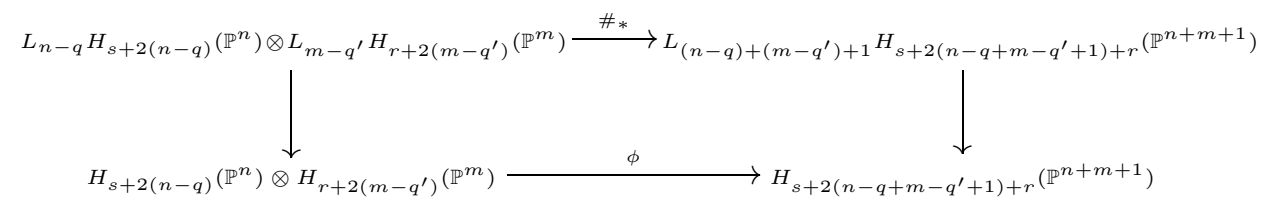

where we define $\phi$ on the generators and we extend it bilinearly: namely, if the cycle classes of the planes $\Lambda_{1} \in H_{2 a+2(n-q)}\left(\mathbb{P}^{n}\right)$ and $\Lambda_{2} \in H_{2 b+2\left(m-q^{\prime}\right)}\left(\mathbb{P}^{m}\right)$ are generators of the corresponding groups, then $\phi\left(\left[\Lambda_{1}\right] \otimes\left[\Lambda_{2}\right]\right)=\left[\Lambda_{1} \# \Lambda_{2}\right]$. This last class is again a generator of $H_{2(a+b)+2(n-q)+2\left(m-q^{\prime}\right)+2}\left(\mathbb{P}^{n+m+1}\right)$. The vertical F-M maps are isomorphisms by Theorem [2.3, so we obtain that the generator $i_{2 c}$ gets pulled back precisely to the sum of the generators $i_{2 a} \otimes i_{2 b}$ with $a+b=c$.

\section{Generalizations}

The proof of Theorem 2.6 suggests a generalization of the result. Instead of taking the Chow Form map, we will look at general correspondences. 
Definition 3.1. Let $\Sigma_{k}$ denote the incidence correspondence defined by

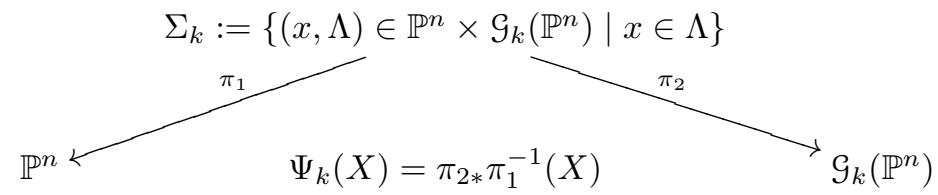

where $X$ is an algebraic cycle and $\pi_{2 *}$ is the push-forward map induced in the level of algebraic cycles by the proper map $\pi_{2}$ (see 12 for a definition and properties).

The map $\pi_{1}$ is flat, and the map $\pi_{2}$ is proper (see [8]). Therefore $\Psi_{k}$ is a well defined map of cycle spaces

$$
\Psi_{k}: \mathcal{C}^{p}\left(\mathbb{P}^{n}\right) \rightarrow \mathcal{C}^{p-k}\left(\mathcal{G}_{k}\left(\mathbb{P}^{n}\right)\right),
$$

(see [12] for a proof of the continuity of the maps induced in the cycle spaces).

Notice that the incidence correspondence which defines the universal quotient bundle is $\Sigma_{p-1}$.

With this notation we have the following.

Theorem 3.2. The map

$$
\Psi_{k}: Z^{p}\left(\mathbb{P}^{n}\right) \rightarrow z^{p-k}\left(\mathcal{G}_{k}\left(\mathbb{P}^{n}\right)\right)
$$

can be represented with respect to the decompositions of the corresponding spaces in the following way:

$$
\prod_{j=0}^{p} K(\mathbb{Z}, 2 j) \stackrel{p}{\longrightarrow} \prod_{r=0}^{p-k} \prod_{\alpha \in H_{2 r}\left(\mathcal{G}_{k}\left(\mathbb{P}^{n}\right)\right)} K(\mathbb{Z}, 2 r)_{\alpha},
$$

where $p$ is the projection into the factors corresponding to the classes $\sigma_{0}, \sigma_{1}, \ldots$, $\sigma_{(p-k)}$.

Proof. The argument follows the proof of Theorem [2.6. We take the homotopy groups and then use the cycle map. In this case we have the following commutative diagram:

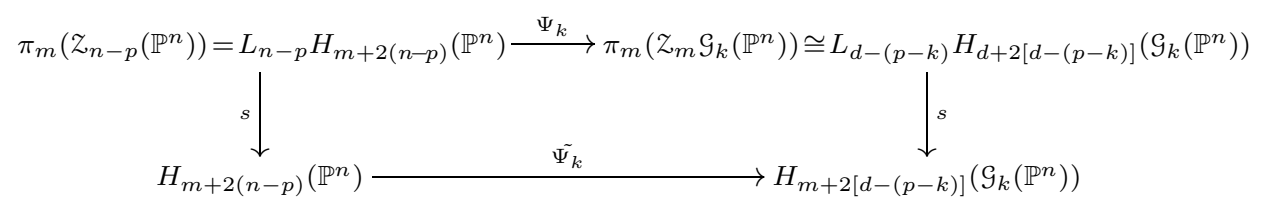

Since the homology of the grassmannian is zero in odd degrees, we are only concerned with the case $m=2 r$. The lower right corner of the diagram imposes the condition $0 \leq 2 r \leq 2(p-k)$. Once again, the definition of the special Schubert cycles implies that if $\Lambda_{2 r+2(n-p)}$ is a plane of dimension $2 r+2(n-p)$ (i.e. the class of a generator of $\left.H_{2 r+2(n-p)}\left(\mathbb{P}^{n}\right)\right)$, then $\tilde{\Psi}_{k}\left(\Lambda_{2 r+2(n-p)}\right)=\sigma_{p-(k+r)}$. 


\section{REFERENCES}

[1] Frederick Justin Almgren Jr., The homotopy groups of the integral cycle groups, Topology 1 (1962), 257-299. MR0146835 (26:4355)

[2] Raoul Bott and Loring W. Tu, Differential forms in algebraic topology, Graduate Texts in Mathematics, vol. 82, Springer-Verlag, New York, 1982. MR658304 (83i:57016)

[3] Eric M. Friedlander and Barry Mazur, Filtrations on the homology of algebraic varieties, Mem. Amer. Math. Soc. 110 (1994), no. 529, x+110. With an appendix by Daniel Quillen. MR.1211371 (95a:14023)

[4] William Fulton, Intersection theory, 2nd ed., Ergebnisse der Mathematik und ihrer Grenzgebiete. 3. Folge. A Series of Modern Surveys in Mathematics [Results in Mathematics and Related Areas. 3rd Series. A Series of Modern Surveys in Mathematics], vol. 2, SpringerVerlag, Berlin, 1998. MR.1644323 (99d:14003)

[5] I. M. Gel'fand, M. M. Kapranov, and A. V. Zelevinsky, Discriminants, resultants, and multidimensional determinants, Mathematics: Theory \& Applications, Birkhäuser Boston, Inc., Boston, MA, 1994. MR 1264417 (95e:14045)

[6] H. Blaine Lawson Jr., Spaces of algebraic cycles, Surveys in Differential Geometry, Vol. II (Cambridge, MA, 1993), Int. Press, Cambridge, MA, 1995, pp. 137-213. MR 1375256 (97m:14006)

[7] Paulo Lima-Filho, On the generalized cycle map, J. Differential Geom. 38 (1993), no. 1, 105-129. MR1231703 (94i:14027)

[8] Joe Harris, Algebraic geometry, Graduate Texts in Mathematics, vol. 133, Springer-Verlag, New York, 1995. A first course, corrected reprint of the 1992 original. MR1416564 (97e:14001)

[9] H. Blaine Lawson Jr. and Marie-Louise Michelsohn, Algebraic cycles, Bott periodicity, and the Chern characteristic map, The Mathematical Heritage of Hermann Weyl (Durham, NC, 1987), Proc. Sympos. Pure Math., vol. 48, Amer. Math. Soc., Providence, RI, 1988, pp. 241263. MR974339(90d:14010)

[10] Eric M. Friedlander and H. Blaine Lawson Jr., A theory of algebraic cocycles, Ann. of Math. (2) 136 (1992), no. 2, 361-428. MR:1185123 (93g:14013)

[11] Judith Plümer, Resultants and the algebraicity of the join pairing on Chow varieties, Transactions of the AMS 349 (1997), no. 6, 2187-2209. MR1407499 (98e:14005)

[12] Eric Friedlander, Algebraic Cycles, Chow varieties, and Lawson homology, Compositio Mathematica 77 (1991), no. 1, 55-93. MR.1091892 (92a:14005)

Max-Planck-Institut für Mathematik, Vivatsgasse 7, D-53111 Bonn, Germany Current address: FEA an MSCI Company, Av. Ricardo Margain \#444, Piso 8, Col. Valle del Campestre, San Pedro Garza Garcia, NL, C.P. 66268, Mexico

E-mail address: llopez@mpim-bonn.mpg.de 